\title{
Interactions with aqueous solutions of the air corona products $\left(^{*}\right)$
}

\author{
J. L. Brisset ( $\left.{ }^{(}\right)$, J. Lelièvre $\left({ }^{2}\right)$, A. Doubla $\left({ }^{1}\right)$ and J. Amouroux ( $\left.{ }^{1}\right)$ \\ (1) Laboratoire des Réacteurs chimiques en phase plasma, E.N.S.C.P., 11 rue Pierre et Marie Curie, 75005 \\ Paris, France \\ (2) Laboratoire de Physicochimie des Solutions, E.N.S.C.P., 11 rue Pierre et Marie Curie, 75005 Paris, \\ France
}

(Reçu le 12 octobre 1989, révisé le 15 janvier 1990, accepté le 13 février 1990)

\begin{abstract}
Résumé. - Le traitement d'une solution aqueuse par un plasma d'air obtenu par décharge couronne pointeplan continue à la pression atmosphérique permet d'aborder en termes de chimie les problèmes d'interaction d'un plasma avec une surface. Une attention particulière est portée aux effets des particules activées non chargées sur la cible qui se manifestent, après filtrage des ions, par une acidification marquée du milieu. L'accroissement d'acidité sous l'effet des entités non chargées (principalement l'oxygène singulet dans des plasmas d'air ou d'oxygène) est mesuré par pH-métrie en fonction de la durée de traitement et de l'intensité du courant électrique : la décroissance linéaire du pH est liée à la quantité d'électricité mise en jeu. De plus, l'immobilisation de solutions basiques d'indicateur dans un gel exposé au flux des espèces neutres activées, permet de révéler l'effet acide. La zone traitée est ainsi mise en évidence; ses dimensions dépendent des paramètres électriques $U, I$, de la distance interélectrodes, de la durée de traitement, de la distance pointecible et de la basicité initiale de la cible. Des abaques sont données pour prévoir les effets du traitement.

Abstract. - The interaction with aqueous solutions of an air plasma provided by a D.C. point-to-plane corona device at atmospheric pressure has been investigated with focusing on the chemical properties of the neutral species after separation from the ion flux. Evidence is given of acid effects induced on the target solution. The increase in acidity due to the treatment by the chargeless activated species - mainly singlet oxygen in air and oxygen plasmas - is measured and related to the quantity of electricity involved in the discharge. In addition, acid-base indicators trapped in a gel are used to develop the acid effect and allow to measure the treated area, in order to get an analytical tool to predict the corona treatment. This area depends on the electric parameters voltage and current intensity, on the distance from the point electrode, on the electrode gap and on the initial acidity of the target.
\end{abstract}

\section{Introduction.}

The corona discharges [1] the interest of which is evident for industrial surface treatments (e.g., copying or elaborating various first layers on materials) are rather easy to operate and can be controlled under smooth conditions (close to NTP). They also may be considered as simplified models for thermal plasmas although the thermal effect be limited to a few degrees raise on the macroscopic scale.

Since we are interested in the chemical effects of the heavy species present in a plasma, we have selected a D.C. point-to-plane corona discharge device as a convenient source of plasma and we have operated it in air at atmospheric pressure. This

(*) This paper was presented at the poster session of the Société Française de Physique Meeting, section B, held in Lyon, 25-29 Sept. 1989. enables us to treat aqueous solutions by the fluxes of ions and of neutral species which are partly raised to an excited state.

The careful examination of the chemical properties of these excited species needs the previous separation of the neutral and charged particles. This separation is obtained by the use of a metallic grid acting as an ion trap as the plane electrode (cf. experimental section), as suggested by Goldman [2]. We could then easily study the chemical properties of the chargeless activated species (CAS), as a first attempt to a systematic investigation, and we started by focusing on the acid-base properties [3-11] which are closely related to corrosion phenomena [8-11].

Preliminary experiments performed with aqueous solutions of acid-base indicators as the targets disposed in various positions with respect to the grid electrode (Fig. 1) gave evidence of the apparition of solvated protons in the solution under exposure to 


\section{ROD}
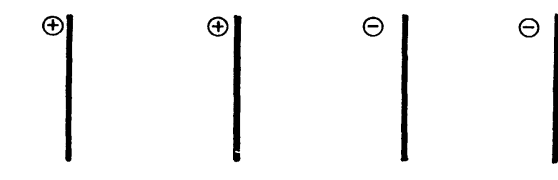

GRID
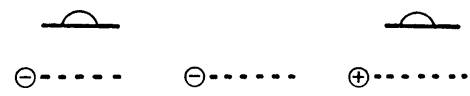

$\oplus \cdots \cdots$

\section{REACTION}

BASE

Fig. 1. - Scheme of preliminary experiments performed on drops of aqueous solutions of acid-base indicators. The point electrode (or rod) is raised to the H.V. and the grid earthed. The liquid target is disposed on a microscope slide. The color change indicates an increased acidity of the solution after treatment by (respectively from left to right) the chargeless species (CAS) and the cations, the CAS, the CAS and the anions, the CAS. Corona discharge performed in atmospheric air.

the discharge which resulted in an acidity increase. It was then concluded [3] on the prominent effects of the chargeless activated species (CAS) over that of the ions. In addition experiments performed with non-aqueous protic solvents (e.g. anhydrous acetic acid) led to similar observations and suggested that the presence of a protic solvent was determinant.

A quantitative investigation of the acidification of the target under exposure to the discharge was then undertaken in the twin purpose of controlling and forecasting the acid effects of the CAS involved in a corona treatment.

\section{Experimental section.}

The general device has been described [3-6] and a scheme of the reactor which is operated in atmospheric air is given (Fig. 2). The 18/10 stainless electrodes are distant from $d(\mathrm{~mm})$ in the range 0$25 \mathrm{~mm}$; the target is disposed at a distance $D>d$
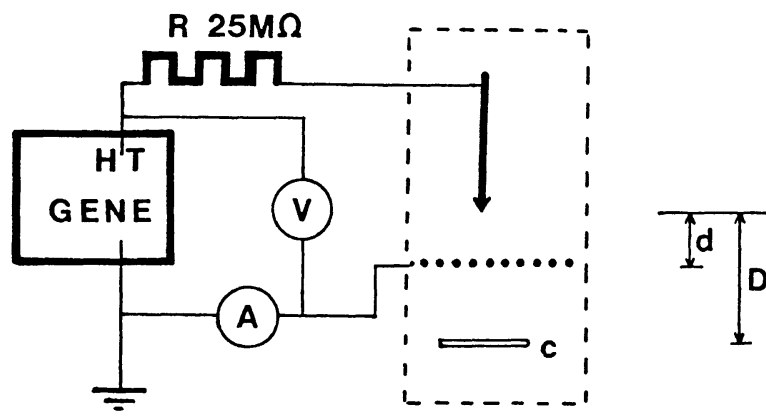

Fig. 2. - General outline of the experimental device for the D.C. point to plane corona discharge. $\mathrm{c}$ is the standard position of the target, at a distance $\mathrm{D}$ from the point electrode. from the point electrode and at the distance $D-d$ below the grid.

The Brandenburg generator supplies a stabilized continuous voltage up to $30 \mathrm{kV}$ between the two electrodes. In all the cases the plane electrode (the grid) is earthed and the point electrode raised to the H.V. (mostly negative).

The target is an aqueous solution of soda. Its acidity is measured ex situ by means of a combined electrode (glass + SCE) adapted to a pH-meter, after long and carefull stirring. When acidity indicators are used, the basic solutions of commercial bromothymol blue (acidity constant 7.1 at ionic strength 0.5 ) are trapped in a $7 \%$ polymethylacrylamide gel to limit evaporation. We prepared the gel according to the general procedure provided by the chemical manufacturer Pharmacia Co. by substituting distilled water to buffers.

\section{Experimental results and discussion.}

1. THE ACIDITY. - According to the Bronsted's definitions, acidity and basicity are related to the proton exchange equilibria in solution between the donor (the acid, e.g., $\mathrm{AH}$ ) and the acceptor (the base, e.g., $\mathrm{A}^{-}$). For example, we have for solvated species :

$$
\mathrm{AH}=\mathrm{A}^{-}+\mathrm{H}^{+}
$$

which is eharaeterized by the value of the Gibbs free energy. The solvent, and in particular amphiprotic solvents such as water, is involved in the equilibrium and acts as acid or base :

$$
\mathrm{AH}+\mathrm{H}_{2} \mathrm{O}=\mathrm{A}^{-}+\mathrm{H}_{3} \mathrm{O}^{+}
$$

which means that two acid-base systems are always needed to react in solution. Hence the acidity of the solution is defined as the decimal cologarithm of the proton activity. The activity is related to the concentration by the activity coefficient which may be calculated from the Debye-Hückel theory relevant to dilute solutions.

It is then evident that, since specific solvation phenomena occur in the concerned equilibria, the values of the free energy will depend on the nature of the liquid medium. It is then impossible to relate the acidity scales in two solvents without referring to some extrathermodynamic assumption in connection with solvation. The same difficulty occurs if one compares solvated and unsolvated systems.

In the gas phase, the species can be considered as unsolvated ; the acidity can be also defined [12], and the free energy is again used as the convenient thermodynamic function. For example, the gas phase acidity of $\mathrm{AH}$ in equilibrium 1 (relevant to unsolvated gaseous species) is given by $\Delta G^{0}(1)$. The gas phase basicity of a species $\mathrm{B}$ involved in a $\mathrm{B} / \mathrm{BH}^{+}$ 
equilibrium is also defined by the relevant free energy. Gas phase acidity and basicity can be calculated from various affinities (proton, hydrogen or electron affinities, resp. PA, HA, EA), binding energies $D$ or ionization potentials IP, if one neglects the entropy terms which remain small.

$$
\begin{aligned}
G A(\mathrm{AH}) & \sim D(\mathrm{AH})+I P(\mathrm{H})-\mathrm{EA}(\mathrm{A}) \\
G B(\mathrm{~B}) & \sim \mathrm{HA}\left(\mathrm{B}^{+}\right)-I P(\mathrm{~B})+I P(\mathrm{H})=\mathrm{PA}(\mathrm{B}) .
\end{aligned}
$$

The proton affinity (Tab. I) appears then as a prominent factor in the calculation of the basicity of a species in the gas phase.

Table I. - Selected Proton Affinity values (kJ.mol ${ }^{-1}$ ) from [12].

\begin{tabular}{|lc|ll|ll}
$\mathrm{NH}_{2}^{-}$ & 1690 & $\mathrm{C}_{6} \mathrm{~F}_{6}$ & 743 & $\mathrm{CH}_{4}$ & 552 \\
$\mathrm{OH}^{-}$ & 1630 & $\mathrm{HCOOH}$ & 718 & $\mathrm{CF}_{4}$ & 527 \\
$\mathrm{~F}^{-}$ & 1550 & $\mathrm{H}_{2} \mathrm{O}$ & 697 & $\mathrm{O}$ & 498 \\
$\mathrm{CN}$ & 1475 & $\mathrm{H}_{2} \mathrm{O}_{2}$ & 678 & $\mathrm{~N}_{2}$ & 494.5 \\
Pyridine & 924 & $\mathrm{CH}_{3} \mathrm{~F}$ & 628 & $\mathrm{HF}$ & 459 \\
Aniline & 876 & $\mathrm{CHF}_{3}$ & 615 & $\mathrm{H}_{2}$ & 424 \\
$\mathrm{CH}_{3} \mathrm{NH}_{2}$ & 876 & $\mathrm{CH}_{2} \mathrm{~F}_{2}$ & 615 & $\mathrm{O}_{2}$ & 422 \\
$\mathrm{NH}_{3}$ & 853.5 & $\mathrm{NF}_{3}$ & 604 & $\mathrm{Ar}$ & 371 \\
$\mathrm{NH}_{2}$ & 782 & $\mathrm{CO}$ & 594 & $\mathrm{~F}$ & 339
\end{tabular}

As already mentioned the correlation between the acidity in the gas phase (involving bare species) and the acidity in solution cannot be done without paying great attention and examples of significant changes are well known (e.g., the order of the basicities of ammonia and the methylamines is different in aqueous solution and in the gas phase).

At first examination the plasma phase should be considered as a gas phase, the involved species as bare or unsolvated particles and their acid or basic character quantified by means of the $G A$ or $G B$ functions. A more practical approach in connection with the industrial treatments leads us to consider that a monolayer of water is adsorbed on a number of plasma treated materials. Such an approach is strongly backed up by the corrosion studies performed on aluminum foils submitted to a corona discharge [8]. We then found more demonstrative to treat aqueous solutions by the discharge and consider water saturated air as the gas phase. We could then observe in the liquid phase the evolution of the solution due to the corona discharge and measure the effects by means of analytical methods adapted to solution chemistry. The data reported here are relevant to the aqueous phase treated by the chargeless activated species of the discharge and appear as a picture of the chemical effects of the neutral species of the plasma phase.

2. QuANTITATIVE pH MEASUREMENTS. - When the discharge is ignited, ions and chargeless activated species are formed and drift in the electrode gap.
The nature of the reacting ions and the relevant clusters has been recently examined in papers [10, $11,13]$ with amphasize on the determinant role of the carbonates, nitrites, nitrates and oxygen anions $\left(\mathrm{O}_{x}\right)^{-}$for negative corona discharges and on that of protons, oxygen $\left(\mathrm{O}_{x}\right)^{+}$and $\left(\mathrm{NO}_{x}\right)^{+}$cations in positive corona discharges.

The chargeless species of chemical importance in the corona discharge in air are reported to be mainly nitrogen oxides and also $\mathrm{CO}_{2}[10,14-16]$ since the occurrence of carbonates in the corrosion products may be related to the presence of about $300 \mathrm{ppm}$ of carbon dioxide in atmospheric air; water vapour (and the relevant clusters) has also to be considered for natural air discharges additionnaly with the $\mathrm{O}_{3}$ molecules formed when the discharge is ignited.

The acidity of small volumes of soda solutions (e.g., $3.5 \mathrm{ml}$ ) is measured ex situ after a vigourous stirring and exposure to the CAS of the discharge. For a given electric intensity, the $\mathrm{pH}$ of the solution is a linear decreasing function of the exposure time (Fig. 3). As evident from figure 3, the slopes directly depend on the current intensity and trend to a limiting value which may be related both to diffusion phenomena into the liquid and to electric conditions (e.g., the transition to the arc). The observed acidity increases which is a logarithmic function of the intensity and the treatment time :

$$
\Delta \mathrm{pH}=-k \cdot I \cdot t=-k \cdot q
$$

gives evidence that the quantity of electricity $q$ is the key parameter involved in the plasma reaction. This variation law also suggests that an equilibrium takes place between solvated protons in the gas phase and in solution, and a scheme of the relevant model has already been considered [5].

The influence of evaporation may also be considered. However it is likely negligible in our case where $\mathrm{pH}$ variations of several units are observed, since it must be reminded that on the mere evaporation statements a $\mathrm{pH}$ variation of 1 unit induces a volume decrease of $90 \%$ which is easily noticeable.

Investigations should be carried on to determine which reactions take place between the chargeless species of the plasma to form solvated protons. By treating an aqueous solution by the flux of the chargeless species created by the corona discharge in air, we limited our study to the chemical effect induced by the CAS passing through a water saturated air layer.

The logarithmic law, $\Delta \mathrm{pH}=-k \cdot q$ must be compared to the results $[17,18]$ relevant to other plasma treatments performed on aqueous solutions, such as the exchange of the ligand $\mathrm{L}$ by $\mathrm{CO}$ in the complexes $\mathrm{Fe}(\mathrm{CN})_{5} \mathrm{~L}$. At this juncture it was found that the decimal logarithm of the concentration of the resultant complex $\mathrm{Fe}(\mathrm{CN})_{5} \mathrm{CO}$ was also a linear function 


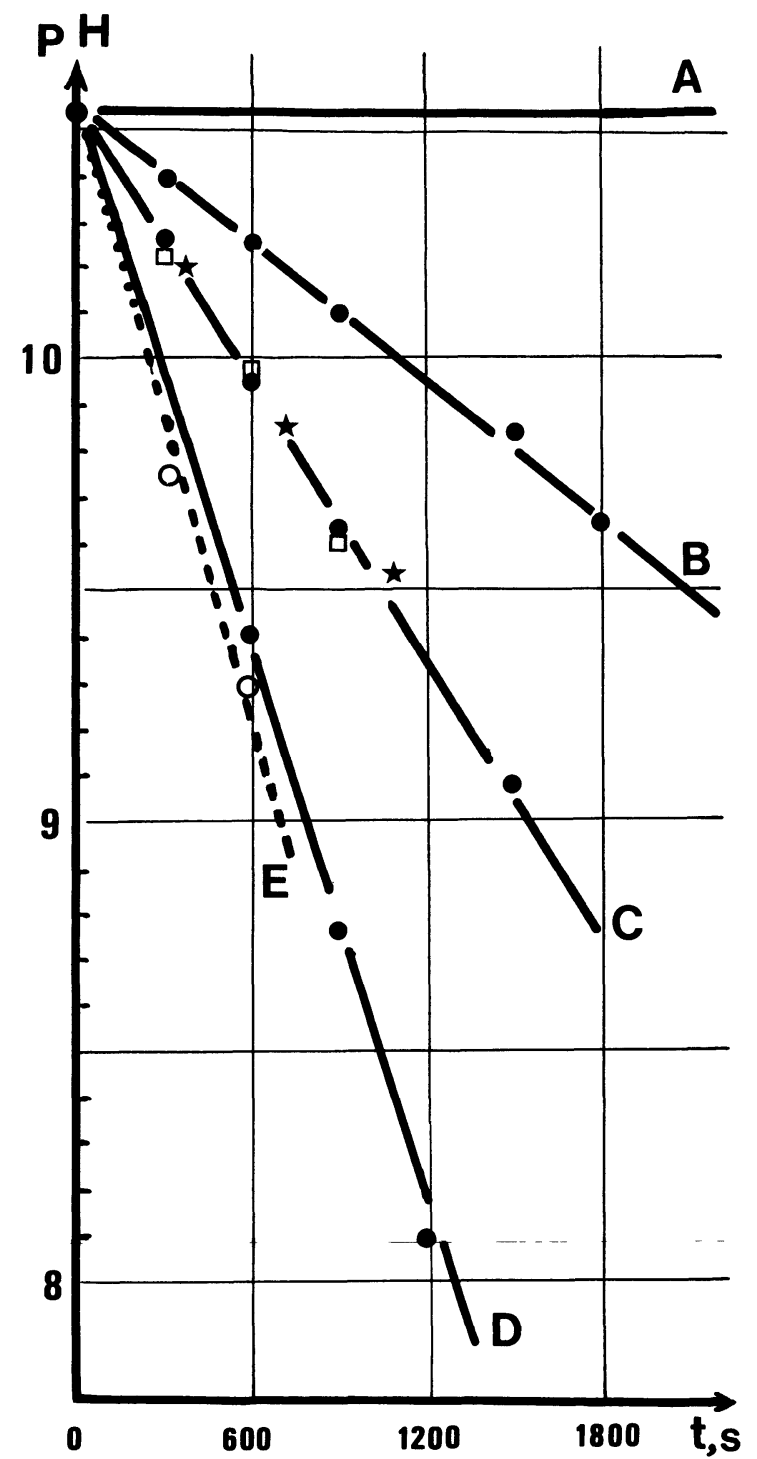

Fig. 3. - Variation of the acidity of the solution $(7.5 \mathrm{ml})$ under exposure to the CAS of the discharge at a fixed distance of the active electrode $(D=17.4 \mathrm{~mm})$. A) untreated solution; $\quad$ B) $I=15 \mu \mathrm{A}, \quad U=3.5 \mathrm{kV}$; C) $I=30 \mu \mathrm{A}, \quad U=4.75 \mathrm{kV}, \quad d=4 \mathrm{~mm}, \quad D=17.4 \mathrm{~mm}$ (filled points) ; $I=30 \mu \mathrm{A}, U=6.7 \mathrm{kV}, \quad d=8 \mathrm{~mm}$, $D=21.4 \mathrm{~mm} \quad$ (stars) ; $\quad I=30 \mu \mathrm{A}, \quad U=7.9 \mathrm{kV}$, $d=12 \mathrm{~mm}, \quad D=25.4 \mathrm{~mm} \quad$ (squares); D) $I=60 \mu \mathrm{A}$, $U=6.5 \mathrm{kV}, d=4 \mathrm{~mm}, D=17.4 \mathrm{~mm}$ (filled points); E) $I=120 \mu \mathrm{A}, U=8.9 \mathrm{kV}, d=4 \mathrm{~mm}, D=17.4 \mathrm{~mm}$ (empty points).

of the quantity of electricity $q$ involved in the discharge, which suggests that the quantity of activated $\mathrm{CO}$ generated by the discharge might also be an exponential function of $q$.

Up to now, we focused on the acid-base effects associated to the treatment by the CAS and we did not consider the effects in connection to the treatments by the ions. Preliminary $\mathrm{pH}$ measurements on aqueous targets electrically isolated and exposed to the single ion fluxes after filtration of the CAS demonstrated that a negative discharge induces a noticeable increase of the solution $\mathrm{pH}$. Although our improved knowledge of the acidification process remains limited, we are now in the position of providing a better understanding of some experimental results reported in [10] with a striking lack of comments and relevant to the treatments of aqueous solutions either by the ions alone or by both the ions and the CAS.

According to [10], treatments of water of the same duration by the ions alone induces very limited $\mathrm{pH}$ changes (i.e., a $0.25 \mathrm{pH}$ increase for a positive corona and a $0.25 \mathrm{pH}$ decrease for a negative corona). When the solution is exposed to the ion and the CAS fluxes, a negative corona discharge induces a significant $\mathrm{pH}$ decrease by $2 \mathrm{pH}$ units while a positive discharge induces a $\mathrm{pH}$ decrease close to $1.5 \mathrm{pH}$ unit within one hour exposure.

The discrepancy with our preliminary results can be mainly attributed to a different experimental procedure, since in our experiments, the target solution is isolated while in [10] the low field electrode is immersed in the solution and induces the classical oxidation-reduction reactions with water when the discharge is ignited. For a positive discharge as used in [10] the reduction of water occurs at the immersed electrode (e.g., in neutral medium : $2 \mathrm{H}_{2} \mathrm{O} \rightarrow \mathrm{H}_{2}+2 \mathrm{OH}^{-}-2 \mathrm{e}^{-}$) and leads to a $\mathrm{pH}$ rise. Similarly for a negative discharge, oxidation of water at the immersed electrode (e.g., $2 \mathrm{H}_{2} \mathrm{O} \rightarrow$ $\mathrm{O}_{2}+4 \mathrm{H}^{+}+4 \mathrm{e}^{-}$in neutral medium) leads to a $\mathrm{pH}$ decrease of unbuffered media.

On the statements of the occurrence of an electrolysis process involving the convenient reactions in the liquid phase and of the prominent acidifying effect of the CAS with respect to the gaseous cations in a positive discharge, the $\mathrm{pH}$ variations reported in [10] may be summarized as in table II. They result of three effects which must be considered: the treatment by the ions and that by the CAS in the gas phase, and the electrode reactions in the liquid phase. A good qualitative agreement is then evident between the experimental data reported in [10] and the predictive considerations developped above.

It is then possible to develop a semi-quantitative approach by assigning arbitrary numbers (which are however proportional to the observed $\mathrm{pH}$ variations) to the treatment by each species alone. We selected -2 and +2 for the cations and anions alone in the gas phase, and -6.5 for the neutrals. For the immersed electrode, the cathode is assigned by +2.5 and the anode by -2.5 . All these numbers are mentioned between brackets in table II, which allows suitable combinations. The comparison with the reported results [10] is then easy and limited $\mathrm{pH}$ changes are found in the balances relevant to the discharge treatments performed with the elimination of the neutral species. The prominent influence of 
Table II. - Evolution of the $\mathrm{pH}$ of distilled water exposed to the point-to-plane corona discharge according to [10].

\begin{tabular}{|c|c|c|c|c|}
\hline $\begin{array}{l}\text { Point electrode } \\
\text { Polarity in the gas phase }\end{array}$ & + & & & - \\
\hline $\begin{array}{l}\text { Prominent Diffusing } \\
\text { Species in the gap }\end{array}$ & $\begin{array}{l}\text { Cations } \\
\text { alone }\end{array}$ & \multicolumn{2}{|c|}{$\begin{array}{l}\text { Neutrals } \\
\text { alone }\end{array}$} & $\begin{array}{l}\text { Anions } \\
\text { alone }\end{array}$ \\
\hline $\begin{array}{l}\text { Acid-base changes in } \\
\text { solution induced by the } \\
\text { gaseous species }\end{array}$ & $\begin{array}{c}\mathrm{pH}: \searrow \\
{[-2]}\end{array}$ & \multicolumn{2}{|c|}{$\begin{array}{l}\mathrm{pH}: \searrow \\
{[-6.5]}\end{array}$} & $\begin{array}{c}\mathrm{pH}: \nearrow \\
{[+2]}\end{array}$ \\
\hline $\begin{array}{l}\text { Immersed electrode } \\
\text { Polarity }\end{array}$ & - & & & + \\
\hline Electrode reaction & $\begin{array}{l}\mathrm{pH}: \nearrow \\
{[+2.5]}\end{array}$ & & & $\begin{array}{l}\mathrm{pH}: \searrow \\
{[-2.5]}\end{array}$ \\
\hline $\begin{array}{l}\text { Balanced } \mathrm{pH} \text { changes } \\
\text { in solution by: }\end{array}$ & $\begin{array}{l}\text { Cations } \\
\text { alone }\end{array}$ & $\begin{array}{l}\text { Cations } \\
+ \text { Neutrals }\end{array}$ & $\begin{aligned} & \text { Anions } \\
+ & \text { Neutrals }\end{aligned}$ & $\begin{array}{l}\text { Anions } \\
\text { alone }\end{array}$ \\
\hline Predicted Variations & {$[+0.5]$} & {$[-6]$} & {$[-7]$} & {$[-0.5]$} \\
\hline Results from ref. [10] & {$[+1]$} & {$[-6]$} & {$[-8]$} & {$[-1]$} \\
\hline
\end{tabular}

the neutrals in the $\mathrm{pH}$ evolution is also verified, in good agreement with the herein reported results. However we must point out that :

- the numbers relevant to the treatment by the cations alone and the anions alone in the gas phase are opposite (i.e., -2 and +2 ) as well as the numbers affected to the immersed electrode reactions (i.e., +2.5 and -2.5 ), in good agreement with the electrolysis laws.

- the numbers assigned to the immersed electrode are slightly superior to those relevant to the point electrode (respect. 2.5 and 2). This can be related to the assumption that all the ions created at the immersed electrode participate to the $\mathrm{pH}$ change while only the major part of the ions created in the gas phase reach the liquid surface and influence the acidity of the solution.

3. ON THE NATURE OF THE CHARGELESS ACTIVATED SPECIES. - Although evidence is given of the interaction with water of the chargeless species generated in a point-to-plane corona discharge which results in an acid raise of the target, we have yet no informations on the nature of the CAS. Since the discharge is realized in air, molecular nitrogen and oxygen appear as the most probable and convenient species to be considered for the origin of the CAS.

Besides the electron distributions of these CAS must be those of a Lewis acid (i.e., a Lewis acid is able to accept on his Lower Unoccupied Molecular Orbital an unshared alectron pair given by a Lewis base) and this situation is verified for singlet oxygen and nitrogen.

In order to precise which species is responsible of the observed acid effect, we made two parts A and B of a soda solution and we dissolved in one of them (sol. A) a large quantity of sodium azotide $\mathrm{NaN}_{3}$ which is a quencher of singlet oxygen. We compared the $\mathrm{pH}$ decrease of the solutions $\mathrm{A}$ and $\mathrm{B}$ after standardized plasma treatments (Tab. III).

In air, the $\mathrm{pH}$ of solution, B decreases by $2.3 \mathrm{pH}$ units while that of solution A decreases only by $1.2 \mathrm{pH}$ unit. This result may be explained in assuming that $\mathrm{NaN}_{3}$ effectively acts as a $\mathrm{O}_{2}(1 \Delta g)$ quencher, which limits the number of reacting CAS and hence the acid effect.

We resumed the experiment with an oxygen plasma and we observed for the same treatment conditions a $\mathrm{pH}$ decay of $0.5 \mathrm{pH}$ unit for solution $\mathrm{A}$ and 3.2 for solution $B$. These results strongly suggest that singlet oxygen has a definite influence on the acidification process.

4. USE OF INDICATORS AS A DEVELOPPER OF THE TREATED AREA. - The reported study allows us to quantify the acid effect due to the chargeless species in the plasma treatment of a certain volume of solution. We were then interested in determining the area treated by the CAS in a corona discharge, and 
Table III. - Acidity increase (pH decay) for the CAS treatments in air or in oxygen of aqueous solutions of indicator with or without singlet oxygen quencher.

\begin{tabular}{|c|c|c|c|c|c|}
\hline $\begin{array}{l}\text { Gaseous } \\
\text { Medium }\end{array}$ & Solution & $\begin{array}{r}\mathrm{p} \\
\text { initial }\end{array}$ & final & $\Delta \mathrm{pH}$ & $\begin{array}{l}\text { Neutral species } \\
\text { involved in pH decay }\end{array}$ \\
\hline AIR & $\begin{array}{c}\mathrm{OH}^{-}+\mathrm{BBT} \\
\mathrm{OH}^{-}+\mathrm{BBT}^{-} \\
+\mathrm{NaN}_{3}\end{array}$ & $\begin{array}{r}9.70 \\
10.30\end{array}$ & $\begin{array}{r}7.40 \\
9.10\end{array}$ & $\begin{array}{l}2.30 \\
1.20\end{array}$ & $\begin{array}{c}\mathrm{N}_{2}+\mathrm{O}_{2}(1 \Delta \mathrm{g}) \\
\mathrm{N}_{2}(1 \quad \Delta \mathrm{g})\end{array}$ \\
\hline $\mathrm{O}_{2}$ & $\begin{array}{c}\mathrm{OH}^{-}+\mathrm{BBT} \\
\mathrm{OH}^{-}+\mathrm{BBT} \\
+\mathrm{NaN}_{3}\end{array}$ & $\begin{array}{r}9.70 \\
10.30\end{array}$ & $\begin{array}{l}6.50 \\
9.80\end{array}$ & $\begin{array}{l}3.20 \\
0.50\end{array}$ & $\mathrm{O}_{2}(1 \Delta \mathrm{g})$ \\
\hline
\end{tabular}

we developped an analytical tool to control the discharge effects. For that purpose, we trapped in a $0.5 \mathrm{~mm}$ thick gel layer a basic solution of acid-base indicator (the bromothymol blue) to limit evaporation, and we exposed it to the CAS. Due to the treatment, a circular spot developped on the gel along the axis of the point electrode, and its colour changed from blue (the base form of the indicator) to yellow (the acid form). The diameter $\varnothing(\mathrm{mm})$ of the spot depends on various parameters which include the electric characteristics of the discharge, the nature of the plasmagen gas and the geometrical parameters of the reactor.

Among the " electrical parameters » which control the discharge and affect $\varnothing$, one finds the voltage difference $U(\mathrm{kV})$ between the electrodes, the intensity $I(\mu \mathrm{A})$ of the current and the exposure time $t(\mathrm{~s})$.

The "geometrical parameters" of the reactor, i.e., the electrode gap $d(\mathrm{~mm})$ and the point-totarget distance $D(\mathrm{~mm})$ also allow to control the flux of the reacting CAS.

Under the label of "chemical parameters", we gather informations relevant to the selected indicator and the acidity level of the target before exposure.

We shall now examine the influence of these groups of parameters on $\varnothing$.

\section{Influence of $U, I$ and $t$ on the diameter $\varnothing$ of the acid spot.}

For a given configuration of the reactor, i.e., for a fixed electrode gap $d$, the diameter of the acid spot is a increasing function of the voltage $U$, the intensity $I$ and the exposure time $t$. In particular, $\varnothing$ varies as $\sqrt{I}$ and $\sqrt{t}$. This result is consequent with the definite influence of the quantity of electricity involved in the discharge and must be paralleled to the electrolysis laws. Besides, the values of $\varnothing$ present a maximum, as it will be examined later, which is a linear function of $I$.
Influence on $\varnothing$ of the point-to-target distance (or observation distance) $D$.

By varying the observation distance $D$ and keeping constant all the other parameters, we could observe that the diameter of the acid spot presents a maximum as $D$ increases. The graph $\varnothing v s$. $D$ (Fig. 4) is the envelope of the acid spots and appears as a longitudinal profile of the surfaces treated by the discharge and may be characterized by the maximum diameter $\varnothing_{\text {max }}$ and the maximum distance to which the acid effeet can be observed : this particular value of $D$ referred to as the observation threshold, $L$.

Such a graph can be drawn for various sets of the electric parameters $U, I$. Figure 4 illustrates a salient propertie of these graphs which are all relevant to the same electrode gap : a homothetical relation is evident between the graphs the characteristic parameters of which may be related to the current intensity since we have :

$$
I_{1} / I_{0}=\varnothing_{\max _{1}} / \varnothing_{\max _{0}}=L_{1} / L_{0} .
$$

An interesting consequence from the analytical point of view is that the observation threshold $L$ is directly related to the intensity, so that the threshold where the acid effect of the discharge is detected can be predicted for given current intensity, electrode gap, selected indicator and initial acidity of the target (Fig. 5).

\section{Influence of the electrode gap $d$ on the detection threshold $L$.}

For given chemical parameters, i.e., fixed indicator and initial acidity of the target, and for a fixed value of the current intensity, the detection threshold $L$ is a decreasing function of the electrode gap $d$ (Fig. 6). This means that small values of the gap are associated (all other parameters being fixed) to important values of the detection threshold, or that the acid 


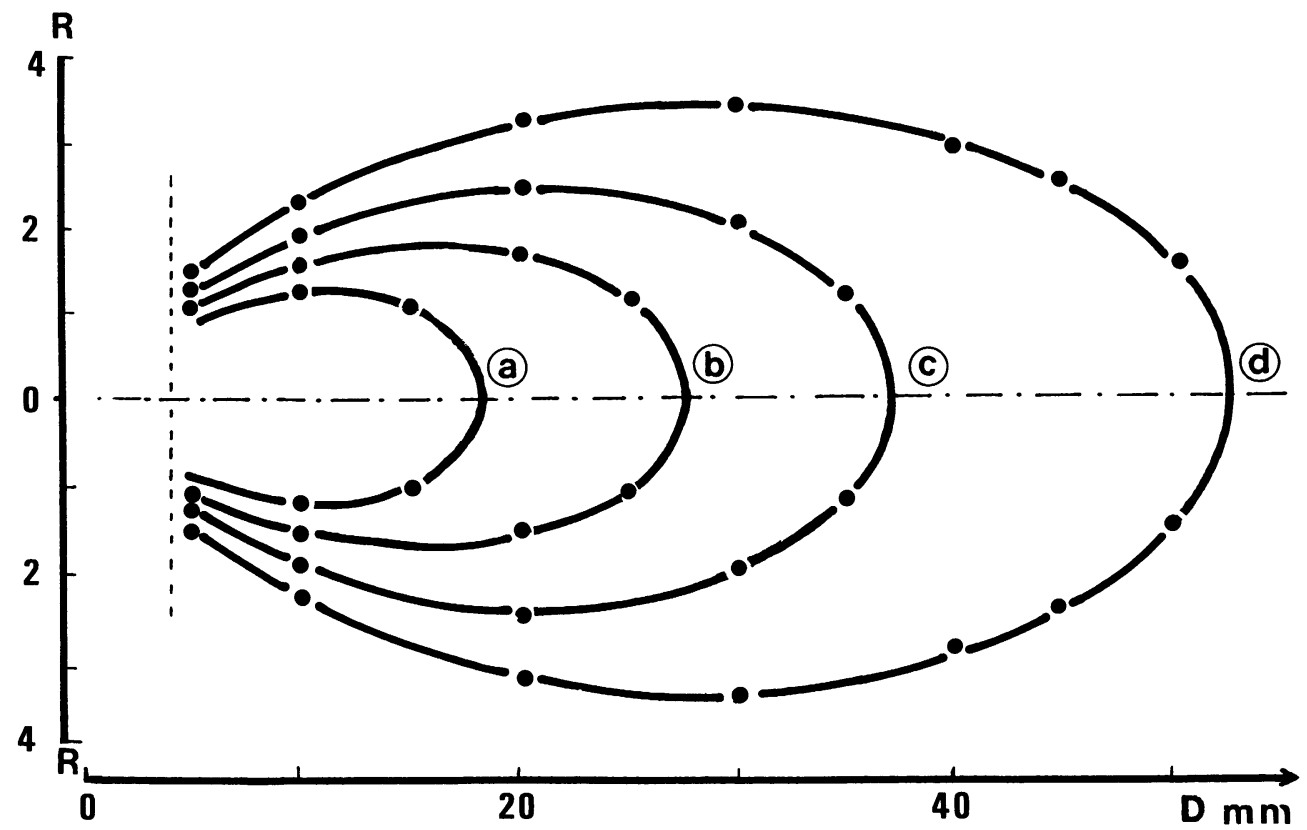

Fig. 4. - Typical plots of the diameter $\varnothing$ of the acid spot $v$ s. the observation distance $D$ mm for an electrode gap $d=4 \mathrm{~mm}$ and various intensities $a$ to $d$ (resp. $60 \mu \mathrm{A}, 90 \mu \mathrm{A}, 120 \mu \mathrm{A}, 170 \mu \mathrm{A}$ ). This plot allows to determine the observation threshold $L$ in particular experimental conditions.
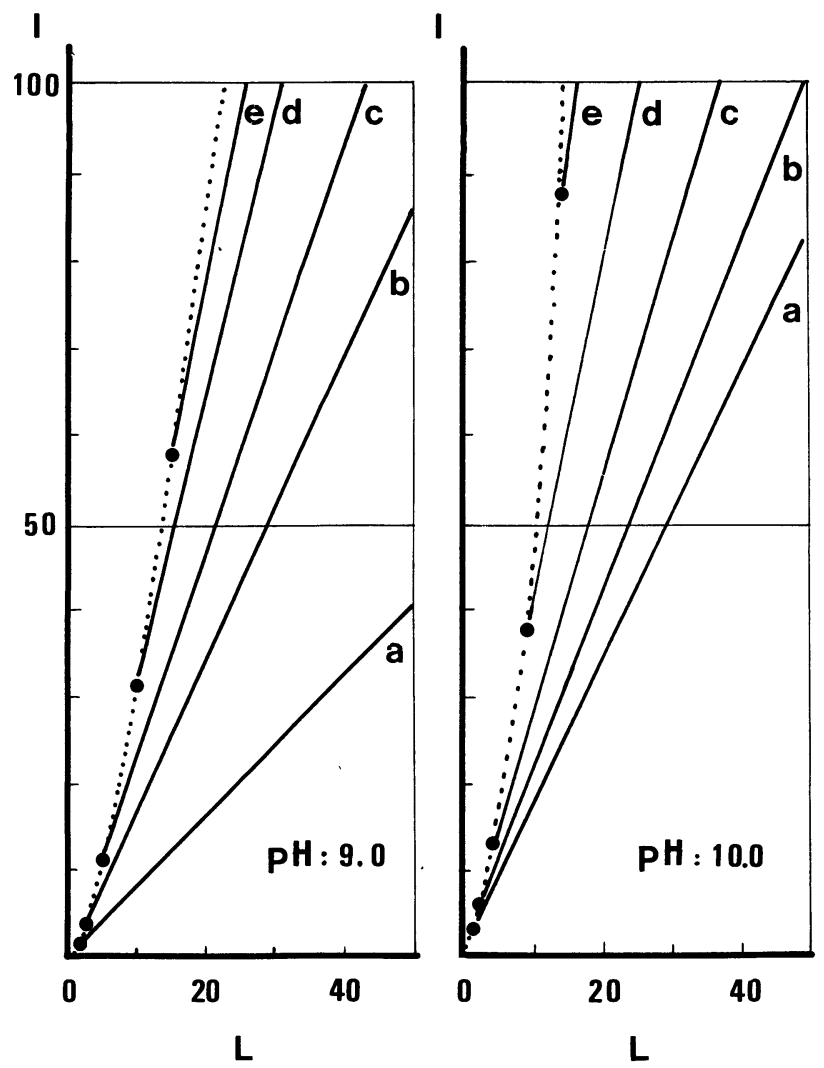

Fig. 5. - Correlations between the current intensity $I$ $(\mu \mathrm{A})$ and the observation threshold $L(\mathrm{~mm})$ for various electrode gaps $a$ to $e$, respectively $2 \mathrm{~mm}, 3 \mathrm{~mm}, 5 \mathrm{~mm}$, $10 \mathrm{~mm}, 15 \mathrm{~mm}$, and two selected acidities of the target $\mathrm{pH}: 9.0$ and 10.0 .

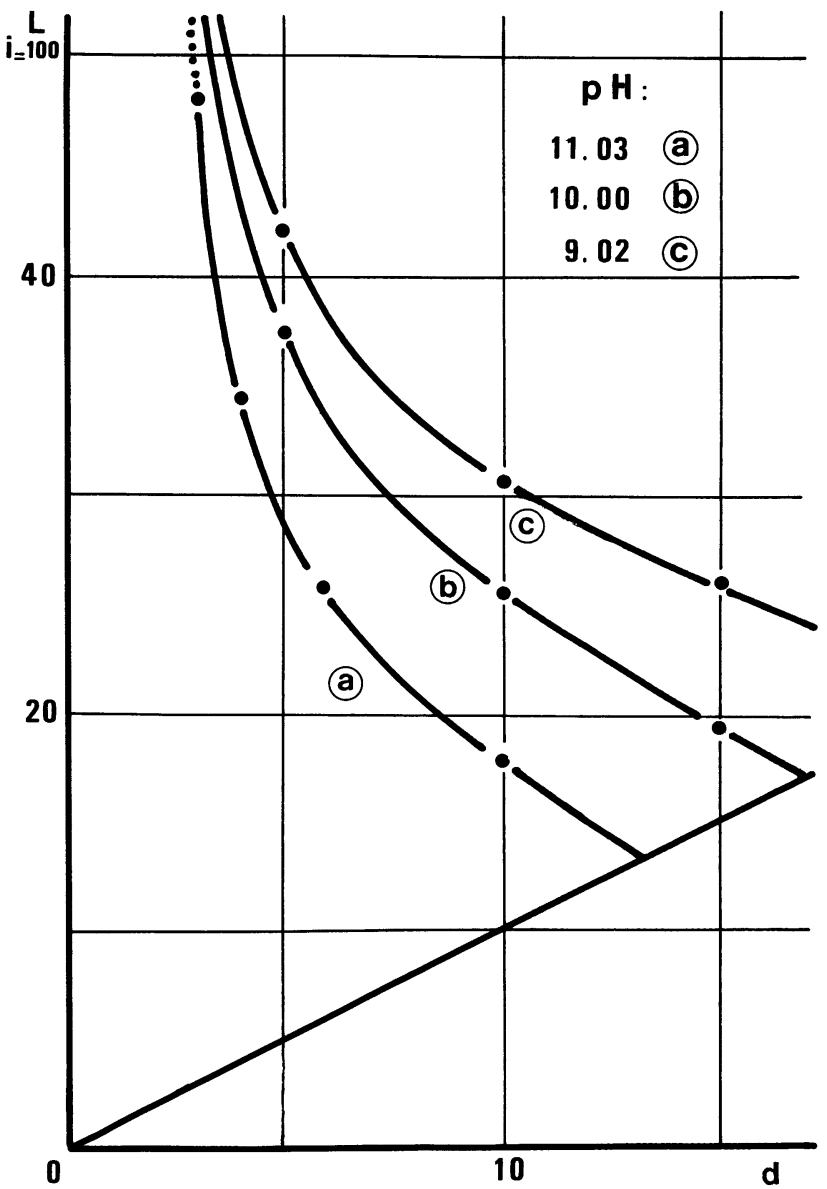

Fig. 6. - Variations of the observation threshold $L(\mathrm{~mm})$ with the electrode gap $d(\mathrm{~mm})$ for a standard intensity $I=100 \mu \mathrm{A}$ and various acidities of the target. 
effect of the CAS can be detected up to several centimeters in these conditions. On the other way, the acid effect associated to the discharge must not be expected (with respect to given chemical parameters) for too large gaps, for a given value of the current intensity.

\section{Influence of the chemical parameters.}

We considered as the chemical parameters the nature of the selected indicator and the initial acidity of the target.

The use as the solution target of an acid-base indicator different from the Bromothymol blue (BTB) induces no change in the qualitative observations of the effects of the treatment by the CAS. A circular spot develops on the gel along the axis of the point electrode, and its colour is that of the acid form of the indicator used. Inferences similar to those developped for BTB are drawn from the observations relevant to the new indicator.

However the diameter of the acid spot depends on the acidity constant of the acid-base indicator for given discharge conditions. A "basic» indicator characterized by a high pKa leads to much higher values of $\varnothing$ than an " acid » indicator with low pKa. This property is easily related to the $\mathrm{pH}$ difference between the acidity of the starting solution $\left(\mathrm{pH}_{0}\right)$ and the acidity constant of the indicator, since the colour change is achieved when the $\mathrm{pH}$ falls from $\mathrm{pH}_{0}$ to a value close to $\mathrm{pK}_{\mathrm{a}}-1$ in the case of acidification.

The influence of the initial acidity of the target was studied with BTB as the standard indicator. We observed for given treatment conditions that the higher values of both the observation threshold $L$ and $\varnothing_{\text {max }}$ correspond to the lower initial $\mathrm{pH}$ value of the target. This is consequent with the above discussion on the $\mathrm{pH}$ differences : a high $\mathrm{pH}_{0}$ value corresponds to a $\mathrm{OH}^{-}$concentrated target solution, and a large $\mathrm{pH}$ decrease from $\mathrm{pH}_{0}$ to $\mathrm{pK}_{\mathrm{a}}-1$ is related to the neutralization of a great number of $\mathrm{OH}^{-}$ions in a small volume of solution, which is connected to a small value of $\varnothing$.

The evolution of $L$ with $\mathrm{pH}_{0}$ follows that of $\varnothing_{\text {max }}$ : for given electrode gap and intensity, the values of $L$ decrease as $\mathrm{pH}_{0}$ increases. The initial acidity of the target also affects the variations of $L$ (relevant to a given current intensity) with the electrode gap $d$, as illustrated by figure 5 . It clearly appears that a given threshold $L$ (for $I$ fixed) is related to increasing values of the electrode gap and the initial basicity of the target (decreasing $\mathrm{pH}_{0}$ values).

\section{Conclusions.}

The study presented is extensively devoted to the acid effect induced in an aqueous solution by the chargeless activated species (CAS) of a point-toplane corona discharge operated in atmospheric air. The quantitative measurements reported give evidence that the quantity of electricity involved in the discharge governs the number of protons created in solution and complementary experiments strongly suggest that singlet oxygen takes a prominent place in the acidification process.

These results were used to elaborate an analytical tool to characterize the discharge by means of the associated acid effects. By using a basic solution of acid-base indicator trapped in a gel and exposed to the CAS, we are now able to predict the surface treated by the flux of the chargeless species in given discharge conditions, and the influences of the selected indicator and that of the initial acidity of the target on the treated area.

\section{Acknowledgments.}

We acknowledge CNRS-PIRSEM for financial support.

\section{References}

[1] Goldman M., Goldman A., Sigmond R. S., Pure Appl. Chem. 57 (1985) 1353-1362.

[2] Goldman M., personal communication.

[3] Brisset J. L., Doubla A., Amouroux J., Proc. Int. Sympos. Plasma Chem. ISPC-8 (Tokyo, Japan, 1987) 1793-1798.

[4] Brisset J. L., DOUbla A., Lelièvre J., Amouroux J., Goldman M., Appl. Surf. Sci. 36 (1989) 530-538.

[5] Brisset J. L., Doubla A., Lelièvre J., Amouroux J., Bull. Soc. Chim. Fr. 615-619 (1989).

[6] Brisset J. L., Doubla A., Lelièvre J., Amouroux J., Analusis 18 (1990) to be published.
[7] BRisset J. L., Doubla A., LelièvRe J., Amouroux J., Proc. Int. Symp. Plasma Chem. ISPC-9 (Bari, Italy, 1989) 550-554.

[8] Goldman A., Sigmond R. S., J. Electrochem. Soc. 132 (1985) 2842-2853.

[9] Brisset J. L., Doubla A., Lelièvre J., Amouroux J., Proc. Int. Symp. Plasma Chem. ISPC-8 (Tokyo, Japan, 1987) 781-786.

[10] Goldman A., Goldman M., Sigmond R. S., Proc. 19th Int. Conf. Phenomena in Ionized Gases (Belgrad, Yugoslavia, 1989) invited paper.

[11] Goldman A., Goldman M., Sigmond R. S., SigMOND T., Proc. Int. Symp. Plasma Chem. ISPC9 (Bari, Italy, 1989) 1654-1658. 
[12] Aue D. H., Bowers M. T., Gas Phase Chemistry Vol. 2. M. T. Bowers Ed. (Academic, New York, 1979) pp. 2-51.

[13] De Vries C. A. M., De Hoog F. J., Schramm D. C., Proc. Int. Symp. Plasma Chem. ISPC-6 (Montreal, Canada, 1983) 317-321.

[14] Peyrous R., Proc. 8th Int. Conf. on Gas Discharges and their applications (Oxford, U.K., 1985) 489492.
[15] Carballeira M., Carballeira A., Gal J. Y., Proc. 14th Conf. on Electric Contacts (Paris, France, 1988) 239-245.

[16] Bourgeois L., Gal J. Y., Carballeira M., To LOSA H., Actes Journées d'Electrochimie 88 (Montpellier, France, 1988) A-8-11.

[17] Doubla A., Thèse de Doctorat ; Université P. et M. Curie, Paris (1989).

[18] Doubla A., Brisset J. L., Amouroux J., J. Chim. phys. to be published. 